\title{
Zur Frage der Aufmerksamkeit
}

\section{Anne-Françoise Allaz}

Prof. Dr. med., Mitglied der Redaktion

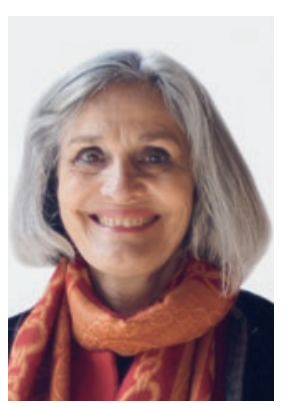

Die jüngste Forderung nach einer «Ökologie der Aufmerksamkeit" [1] in der Medizin, die das Ziel verfolgt, durch ein verbessertes Arbeitsumfeld ein konzentrierteres und aufmerksameres Arbeiten zu ermöglichen, verdient unsere Beachtung angesichts der zunehmend durch Unterbrechungen und Ablenkungen gekennzeichneten Arbeitsweise der heutigen Ärzteschaft. Eine Studie am Waadtländer Universitätsspital CHUV hat ergeben, dass die dortigen Internistinnen und Internisten innert einer Stunde im Schnitt 15 verschiedene Aufgaben übernehmen [2]! Tatsächlich bemängeln zahlreiche Ärztinnen und Ärzte in Ausbildung die häufigen Ablenkungen durch unterschiedlichste Anforderungen wie die klinische Dokumentation und die Datenerfassung zu Nachweis- oder Fakturierungszwecken. Kurz, durch all die unvermeidlich gewordenen medizinisch-administrativen «kategorischen Imperative», die den Arbeitsalltag der Medizinerinnen und Mediziner in Spitälern und Praxen zerstückeln und erschweren.

"Aufmerksamkeit bei allem ist das, was uns rettet", sagte Bossuet. Damit hatte er zweifelsohne recht, denn wie oft kommt es zu Fehlern infolge von Ablenkung und mangelnder Aufmerksamkeit? Umgekehrt wirkt sich das Vermeiden von Unterbrechungen und Tätigkeitswechseln erwiesenermassen positiv auf das Urteilsvermögen und die Fähigkeit aus, komplexe Aufgaben zu erfüllen. Ausserdem - und dies ist nicht unerheblich - verbessern Aufmerksamkeit und Konzentration die Qualität der Arbeit und machen sie zufriedenstellender und sinnhafter [3].

Aufmerksamkeit ist in der Medizin zweifellos eine Schlüsselkompetenz - egal, ob man sich aufmerksam auf eine Aufgabe oder auf eine Patientin oder einen Patienten konzentriert. Dieser «bewusste und intensive Einsatz des Geistes» ist eine wertvolle Ressource, die erstaunlich häufig sakrale Formen annimmt. "Aufmerksamkeit ist eine Form des Gebets», meint beispielsweise der zeitgenössische laizistische Humanist Claudio Magris. Sie gibt dem Gegenüber - der Patientin oder dem Patienten - das Gefühl, dass man ihm wirklich zuhört. Als Arbeitsinstrument in der Psychoanalyse macht die "freischwebende» oder "gleichschwebende» Aufmerksamkeit es möglich, der Patientin oder dem Patienten mit dem «dritten Ohr» zuzuhören, wie es der österreichische Psychoanalytiker Theodor Reik so treffend ausgedrückt hat.
Angesichts der beunruhigenden Zunahme von «Burnout»-Fällen bei Ärztinnen und Ärzten - die zum Teil auf eine administrative Überlastung zurückgeführt werden [4] - sollten wir die Arbeitsweise in unserem spezifischen Umfeld überdenken. Lassen sich bestimmte Prozesse verbessern? Müssen wir beispielsweise wirklich ständig verfügbar - und somit ablenkbar - sein? Könnte durch eine Optimierung der Informationsübertragung vielleicht deren Häufigkeit verringert werden? Welche administrativen Aufgaben liessen sich delegieren? Sind die Praxis und ihre Abläufe so organisiert, dass ein aufmerksames Arbeiten gefördert wird? Auf diese Fragen lassen sich vielfältige und kreative Antworten finden. Immer mehr Spitäler und Arztpraxen werden sich des Problems bewusst. Einige haben ihre Arbeitsabläufe grundlegend umstrukturiert, um Tätigkeitsunterbrechungen zu reduzieren, wieder mehr Zeit für klinische Aspekte zu haben und damit sowohl die Aufmerksamkeit im Allgemeinen als

\section{"Aufmerksamkeit ist eine Form des Gebets.» (Claudio Magris)}

auch insbesondere die Aufmerksamkeit gegenüber den Patientinnen und Patienten zu fördern [5, 6].

Die Vorteile einer verbesserten Lenkung der Aufmerksamkeit sind unbestreitbar, denn nur wer dem Gegenüber seine volle Aufmerksamkeit schenkt, kann ihm entsprechend dem Kerngedanken der Medizin-wirklich kompetent und authentisch begegnen und somit dazu beitragen, dass wir unseren Beruf auch weiterhin zufriedenstellend ausüben können.

Literatur

1 Kissler MJ, et al. Towards a medical ecology of attention. New Engl J Med. 2021;384:299-301.

2 Mean M, et al. Computer usage and task-switching during resident's working day: Disruptive or not? PLoS ONE. 2017;12(2): e0172878.

3 Crawford MB. The world beyond your head: on becoming an individual in an age of distraction. New York: Farrar, Straus and Giroux; 2015.

4 Shanafelt TD, et al. Relationship Between Clerical Burden and Characteristics of the Electronic Environment with Physician Burnout and Professional Satisfaction. Mayo Clin Proc. 2016; 91:836-48.

5 Darbellay Farhoumand P, et al. Réorganisation interprofessionnelle du travail dans les unités de soins. Projet «Plus de temps pour les patients» aux HUG. Rev Med Suisse. 2018;14:1550-5.

6 Alkalay-Loher M, et al. Das erfolgreiche Beispiel des Kantonsspitals Baselland macht Schule. Lean Bettenstation: die 7 Taktiken zum Erfolg. PDF, 2016. www.walkerproject.com 